\title{
Re-thinking the fixed-criterion model of perceptual decision-making
}

\author{
Jenn Laura Lee, Rachel Denison, and Wei Ji Ma \\ Center for Neural Science and Department of Psychology, New York University
}

April 2, 2021

\begin{abstract}
Perceptual decision-making is often conceptualized as the process of comparing an internal decision variable to a categorical boundary, or criterion. How the mind sets such a criterion has been studied from at least two perspectives. First, researchers interested in consciousness have proposed that criterion-crossing determines whether a stimulus is consciously perceived. Second, researchers interested in decision-making have studied how the criterion depends on a range of stimulus and task variables. Both communities have considered the question of how the criterion behaves when sensory information is weak or uncertain. Interestingly, however, they have arrived at different conclusions. Consciousness researchers investigating a phenomenon called "subjective inflation" - a form of metacognitive mismatch in which observers overestimate the quality of their sensory representations in the periphery or at an unattended location - have proposed that the criterion governing subjective visibility is fixed. That is, it does not adjust to changes in sensory uncertainty. Decision-making researchers, on the other hand, have concluded that the criterion does adjust to account for sensory uncertainty, including under inattention. Here, we mathematically demonstrate that previous empirical findings supporting subjective inflation are consistent with either a fixed or a flexible decision criterion. We further show that specific experimental task requirements are necessary to make inferences about the flexibility of the criterion: 1) a clear mapping from decision variable space to stimulus feature space, and 2) a task incentive for observers to adjust their decision criterion as response variance increases. We conclude that the fixed-criterion model of subjective inflation requires re-thinking in light of new evidence from the probabilistic reasoning literature that decision criteria flexibly adjust according to response variance.
\end{abstract}

\section{Introduction}

We must often make judgments about what we see, from categorizing a person in the distance as a friend or stranger to deciding whether a faint sense of motion in our periphery was really something moving or in fact nothing at all. Such perceptual decisions require that we produce a categorical answer based on the available visual information. Perceptual decision-making is often conceptualized, therefore, as the process of comparing an internal decision variable to a categorical boundary, or criterion.

How human observers set such a criterion has been studied from at least two perspectives. Researchers interested in consciousness tend to study decisions like whether or not a stimulus is present, and they have sometimes conceptualized the detection criterion as a threshold for conscious perception of a stimulus. Meanwhile, researchers interested in decision-making tend to study decisions like whether a clearly visible stimulus is a member of one category or another, and they have generally conceptualized the categorization criterion as a cognitive variable that depends on context and goals. Both groups of researchers have used the same modeling framework - signal detection theory - to investigate their questions of interest. Interestingly, however, they have arrived at different conclusions about the properties of the criterion.

Researchers interested in conscious perception have proposed that the criterion is a fixed threshold [1]-in line with a theoretical perspective that consciousness is all or none and that subjective perception occurs only when the first-order signal crosses a criterion. Proponents of a "higher-order thought" (HOT) theory of consciousness propose that a higher-order representation is needed to make a first-order perceptual state [2]. Importantly, the model favoured by higher-order theorists assumes that the criterion is set "after consideration of the statistical properties of both the internal signals for the attended and unattended stimuli," ([2], p10) positing that the criterion remains at a fixed value regardless of one's level of attention. Evidence for this view has come from the finding that visual phenomenology is often inflated above what would be predicted based on sparse sensory content [3]: observers report peripheral [4] or unattended [1] stimuli as "seen" more often than would be expected based on the observers' performance in discriminating features of those 
stimuli. Signal detection theoretic models of subjective inflation have explained the findings using a fixed or unified criterion, in which peripheral and unattended sensory signals are more variable, reflecting greater uncertainty, and are thus more likely to cross the fixed threshold. Researchers have further proposed that subjective inflation, operating by this mechanism, might underlie the sense that our visual world is uniformly rich despite the poverty of visual information at the periphery and at unattended spatial locations. [3]

Researchers interested in decision-making, on the other hand, have concluded that the criterion is a flexible boundary - in line with a theoretical perspective that perceptual decision-making, even about very simple stimuli, is a cognitive operation that is sensitive to a variety of factors, including prior knowledge, task incentives, and uncertainty. Most relevant to the issue of subjective inflation is the question of whether and how observers incorporate uncertainty information in their perceptual decisions, as both peripheral and unattended stimuli are associated with higher uncertainty compared to foveal and attended stimuli.

Many studies have demonstrated that observers incorporate uncertainty information when integrating prior knowledge with uncertain sensory information (Acerbi, 2014 [5]; Kording, 2004 [6]; Jazayeri, 2010 [7]). The cue combination literature provides further evidence that uncertainty information is represented and used in perceptual decision-making: many studies demonstrate that observers integrate noisy cues from multiple sensory modalities in a near-optimal way, where each cue is weighed in accordance with its reciprocal variance (Alais, 2004 [8]; Kording, 2007 [9]; van Beers, 1999 [10]; Knill, 2003 [11]). Lastly, subjects incorporate uncertainty information in many tasks requiring higher-level visual cognition (Zhou, 2018 [12]); Shen, 2016 [13]; Qamar, 2013 [14]; Ma, 2011 [15]; Yang, 2016 [16]).

In particular, recent work has suggested that observers also incorporate uncertainty when it arises from peripheral viewing (Zhou, 2018 [12]) or inattention (Denison, 2018 [17]). Therefore, decision making researchers have suggested that criteria are flexible for situations very similar to those for which consciousness researchers have suggested that criteria are fixed.

This divergence poses a puzzle: Why have different research communities studying closely related questions and applying similar mathematical models generated different proposals about the flexibility of decision behavior? One possibility is that the experimental results in each community are truly incompatible perhaps, for example, there is a fundamental difference between the detection tasks that consciousness researchers tend to study and the categorization tasks that decision researchers tend to study. Another possibility, however, is that the two sets of results are compatible and the two research traditions could be unified.

Here we argue that a unification is possible. To do so, we focus on two key studies, one from each community, and show mathematically how they can be reconciled. We have chosen to focus on Rahnev (2011) [1] in the consciousness tradition, and Denison (2018) [17] in the decision making tradition, both of which investigated spatial attention. Rahnev (2011) [1] was the original study to provide evidence for subjective inflation under inattention and to explain it with a fixed criterion. Denison (2018) [17] specifically investigated how attention affects decision behavior and found evidence for flexible criteria.

To investigate this apparent difference, we mathematically identified the space of parameter combinations consistent with behavioural evidence used to support the "fixed criterion" hypothesis in Rahnev et al. 2011, and show that this space in fact contains a large set of "flexible criterion" solutions. We further show that the empirical evidence presented by both of these studies is consistent with a broader class of generalized Bayesian observers that take uncertainty into account when setting decision criteria. We conclude that previous proposals that subjective inflation arises from a fixed decision rule are therefore unwarranted; both sets of findings are consistent with a flexible decision criterion that accounts for attention. Finally, we describe how Denison et al. employed two methodological improvements upon the standard signal detection theoretic framework, which allowed inferences about decision rules that were not possible in previous studies - lending strength to the evidence for flexible criterion-setting.

\section{Background}

\section{A. Signal detection theory}

Both conscious perception and uncertainty researchers use a signal detection theoretic modelling framework to investigate their questions of interest. In Signal Detection Theory (SDT), a visual stimulus is internally mapped to a one-dimensional decision variable in the observer's head, corresponding- in theory- to whichever dimension of the stimulus which is task-relevant. In a stimulus detection paradigm, the subject is instructed to respond about whether they believe the stimulus was "present" or "absent" on a given trial. Repeated presentations of the same stimulus (e.g. a Gabor with the same contrast strength) are thought to result in a decision variable with some amount of trial-to-trial variability $(\sigma)$. [18]. A trial with a low decision variable value should be classified as "unseen," and one with a high value should be classified as "seen." Each internal decision variable distribution for "present" or "absent" stimuli is a theoretical distribution formed from infinite repeated observations of stimulus-present or "stimulus-absent" trials. These two decision variable distributions overlap one another, resulting in some degree of inherent ambiguity for any one given observation. The decision-making system resolves this by setting a decision boundary (also called a decision criterion): an observation should be classified as "absent" unless it yields an decision variable value greater than a given decision criterion, in which case it should be classified as "present." If the decision-making system has perfect metacognitive access to the true mean $(\mu)$ and variance $(\sigma)$ of the two decision variable distributions, then it should be able to compute the optimal decision criterion- that is, the threshold at the intersection of these two distributions that 
maximizes decision accuracy over many trials. (Figure 2, panel A)

SDT is a very general framework, and the decision variable can range from a basic sensory signal to a highly derived cognitive quantity. The decision variable is often referred to, abstractly, as the "strength of evidence" for one decision category or another. Green and Swets [18] conceptualized the decision variable as a log posterior ratio, a comparison of the probabilities of each category. To actually calculate such probabilities, one needs a generative model describing how stimulus inputs are transformed into internal responses. In perception science, it is often possible to specify such a generative model, in which a stimulus generates a noisy internal measurement, which can then be used to determine the probability of each stimulus given that internal response. This measurement is "absolute" in the sense that it is considered to be a physical quantity in the brain that is directly related to the stimulus (e.g., neural firing rate or estimated orientation). The fixed criterion proposal refers to the measurement space: the idea is that on each trial observers make a decision by comparing their sensory measurement to some fixed value.

The first step in formalizing a signal detection theoretic model is to define the statistics of the observations. Part of the generative model is defined by the task at hand: the stimulus $s$ takes two discreet values- on a detection task, the stimulus is either present or absent. If the stimulus is present, $s$ has a fixed, experimenterset value, which we will denote by $\mu$. If it is absent, we will define it as 0 . The two stimulus values are equally likely on any given trial, so that

$$
p(s=0)=p(s=\mu)=0.5 .
$$

To complete the generative model, we have to specify the nature of the observations. We assume that on each trial, the observer makes a noisy measurement $x$ of the stimulus $s$. As is standard in signal detection theory (and motivated by the central limit theorem) we assume the noise to be zero-mean Gaussian noise. Thus, we have:

$$
p(x \mid s)=\frac{1}{\sqrt{2 \pi \sigma^{2}}} e^{-\frac{(x-s)^{2}}{2 \sigma^{2}}} .
$$

If the signal (stimulus) is absent, then the decision variable is drawn from a normal distribution with mean 0 and standard deviation $\sigma$. If the signal is present, then the variable is drawn from a normal distribution with mean $\mu$ and standard deviation $\sigma$. The distributions $p(s)$ and $p(x \mid s)$ fully define the generative model.

Let's say that on a given trial, the measurement of the stimulus is $x_{\text {trial }}$ and the observer is asked to infer whether the stimulus is present, i.e. to infer $s$. Given the generative model, the log posterior ratio over $s$ is then

$$
\begin{aligned}
\operatorname{LPR} & =\log \frac{p\left(x_{\text {trial }} \mid s=\mu\right)}{p\left(x_{\text {trial }} \mid s=0\right)} \\
& =\ldots=\frac{\mu}{\sigma^{2}}\left(x_{\text {trial }}-\frac{\mu}{2}\right),
\end{aligned}
$$

which is a multiple of the measurement itself. Therefore, it is, under this generative model, equivalent to use either the log posterior ratio or the measurement as the decision variable, and we choose the latter.

To make a decision, the observer responds "present" when the measurement exceeds the criterion, i.e. when

$$
x_{\text {trial }}>k \text {. }
$$

We will refer to $k$ as the absolute criterion. We also introduce a relative decision criterion $c$, which is a linear transformation of the absolute criterion $k$ :

$$
c=\frac{k-\frac{\mu}{2}}{\sigma}
$$

The absolute criterion has the same units as $s, x, \mu$, and $\sigma$, whereas the relative criterion is dimensionless.

The model observer's sensitivity is a signal-to-noise ratio:

$$
d^{\prime}=\frac{\mu}{\sigma}
$$

A higher $\mu$ (a larger-magnitude difference between "present" and "absent") or a lower $\sigma$ (less measurement noise) will result in less overlap between the "present" and "absent" distributions, and higher sensitivity. The same $d^{\prime}$ could arise from an infinite number of combinations of $\mu$ and $\sigma$.

\section{B. Summary of Rahnev et al.'s experiment}

The Rahnev et al. experiment that we primarily consider consists of a detection task where attention is manipulated with visual cues. The screen is divided into four quadrants. Each quadrant displays either a patch of visual noise, or a noisy Gabor patch. Each diagonal pair contains the same stimulus. Subjects are cued to attend to either diagonal pair. They are then probed to respond about whether they saw or did not see a Gabor, at either the cued or uncued locations. Trials on which the cued locations are probed for response are "valid" trials (on which attentional allocation is presumably high), and trials on which the uncued locations are probed were "invalid trials" (on which attentional allocation is presumably low).

In both valid and invalid conditions, experimenters measured the subjects' hit rate $H$ and false-alarm rate $F$. The authors use z-scores for $H$ and $F$ to compute sensitivity $d^{\prime}$ and relative criterion $c$ according to the standard formulae from signal detection theory:

$$
\begin{aligned}
d^{\prime} & =z(H)-z(F) \\
c & =-\frac{z(H)+z(F)}{2}
\end{aligned}
$$

The authors titrate the contrast of the stimuli such that in approximation

$$
d_{\mathrm{val}}^{\prime}=d_{\mathrm{inv}}^{\prime}
$$


where "val" and "inv" refer to the subsets cue condition (valid or invalid). The empirical finding can then be summarized as

$$
c_{\text {inv }}<c_{\mathrm{val}} \cdot
$$

In words, the relative decision criterion in the invalid condition was measured to be smaller than in the valid condition. Based on this finding, what can we infer about the absolute criterion in either attentional condition? We show in the next section that this finding is insufficient to infer a fixed absolute criterion.

\section{Degeneracy of solutions}

\section{A. Inequality Accounting for Rahnev's Findings (with $d^{\prime}$ matched)}

There are a number of combinations of absolute criteria and noise parameters that are consistent with the empirical findings in Experiment 1 of Rahnev et al. 2011. Each observer measurement is made under one of two conditions: valid (attended) and invalid (unattended). Therefore, we first allow $\mu$ and $\sigma$ to be condition-dependent and correspondingly attach labels "val" and "inv":

$$
\begin{aligned}
& d_{\mathrm{val}}^{\prime}=\frac{\mu_{\mathrm{val}}}{\sigma_{\mathrm{val}}} \\
& c_{\mathrm{val}}=\frac{k_{\mathrm{val}}-\frac{\mu_{\mathrm{val}}}{2}}{\sigma_{\mathrm{val}}} \\
& d_{\mathrm{inv}}^{\prime}=\frac{\mu_{\mathrm{inv}}}{\sigma_{\mathrm{inv}}} \\
& c_{\mathrm{inv}}=\frac{k_{\mathrm{inv}}-\frac{\mu_{\mathrm{inv}}}{2}}{\sigma_{\mathrm{inv}}}
\end{aligned}
$$

We now assume that in the invalid condition, when attention is lower, the level of measurement noise (i.e. the trial-to-trial variance in the observer's decision variable) is higher. In other words, $\sigma_{\mathrm{inv}}>\sigma_{\mathrm{val}}$. Eq. (10) can be reformulated:

$$
\frac{\mu_{\text {inv }}}{\sigma_{\text {inv }}}=\frac{\mu_{\text {val }}}{\sigma_{\text {val }}}
$$

Rahnev's empirical finding, per Eq. (11), can be reformulated as:

$$
\begin{aligned}
& \frac{k_{\text {inv }}-\frac{\mu_{\text {inv }}}{2}}{\sigma_{\text {inv }}}<\frac{k_{\mathrm{val}}-\frac{\mu_{\mathrm{val}}}{2}}{\sigma_{\mathrm{val}}} \\
& \frac{k_{\text {inv }}}{\sigma_{\text {inv }}}-\frac{\mu_{\text {inv }}}{2 \sigma_{\text {inv }}}<\frac{k_{\mathrm{val}}}{\sigma_{\text {val }}}-\frac{\mu_{\mathrm{val}}}{2 \sigma_{\text {val }}}
\end{aligned}
$$

At this point, it is helpful to consider the range of $k_{\text {val }}$. Empirically, Rahnev et al. find that $c_{\mathrm{val}}>0$, which by Eq. 6 implies that $k_{\mathrm{val}}>0$. (In fact, the necessary and sufficient condition for the latter is that $c_{\mathrm{val}}>-\frac{d^{\prime}}{2}$, which is by extension also empirically true.) Henceforth, we will assume that $k_{\text {val }}>0$.

Combining Eqs. (16) and (18), we find

$$
\begin{aligned}
& k_{\text {inv }}<\frac{\sigma_{\text {inv }}}{\sigma_{\text {val }}} k_{\text {val }} \\
& k_{\text {val }}>0
\end{aligned}
$$

Thus, in the signal detection theory model of the task, any combination of $k_{\text {inv }}, k_{\text {val }}>0, \sigma_{\text {inv }}$, and $\sigma_{\text {val }}$ that satisfies Eqs. (19) and (20) can explain the experimental findings for detection tasks. We will refer to Eq. (19) as the Inequality Accounting for Rahnev's Findings, or the IARF for short. Throughout this paper, we will assume that Eq. (20) holds for stimulus detection tasks.

\section{B. Rahnev solution}

The authors next propose a kind of explanatory model, which, following Gorea [19], they call a "unified criterion" model. In this model, the observer uses the same, fixed measurement criterion in both conditions, i.e.

$$
k_{\text {fixed, inv }}=k_{\text {fixed, val }} .
$$

This is one solution to Eq. (19), since we would have

$$
0<k_{\text {fixed, inv }}=k_{\text {fixed, val }}<\frac{\sigma_{\text {inv }}}{\sigma_{\text {val }}} k_{\text {val }} .
$$

\section{All solutions}

Rahnev's fixed-criterion model is far from the only solution to Eq. (19). In particular, $k_{\text {inv }}$ could be different from $k_{\text {val }}$, as long as it is smaller than $\frac{\sigma_{\text {inv }}}{\sigma_{\text {val }}} k_{\text {val }}$. This means that the experimental results are consistent with a wide range of models whose measurement criteria are in fact sensitive to uncertainty. For example, even in the scenario that $\sigma_{\text {inv }}>\sigma_{\text {val }}$, one could imagine an observer who scales their measurement criterion by the square root of the noise level. Then,

$$
k_{\mathrm{sqrt}, \text { inv }}=\sqrt{\frac{\sigma_{\text {inv }}}{\sigma_{\text {val }}}} k_{\text {sqrt, val }}<\frac{\sigma_{\text {inv }}}{\sigma_{\text {val }}} k_{\text {sqrt, val }},
$$

where we take advantage of the fact that the square root of a positive number greater than 1 is smaller than the number itself. Thus, the IARF is satisfied even despite the measurement criterion shifting.

In panel A and B of Figure 1, we visualize three possible relationships between the trial-to-trial variance of the decision variable $(\sigma)$ and an observer's absolute ( $k$, panel A) or relative ( $c$, panel B) decision criterion. Inattention could lead to an increase in the trial-to-trial variability of the decision variable on a detection or discrimination task, i.e. an increase in $\sigma$. Fixed criterion models assume that the absolute criterion remains fixed as $\sigma$ increases (purple line, panel A). This corresponds to a decreasing relative criterion as variability increases (purple curve, panel B)in other words, with fixed criteria, we should expect the relative criterion to be more liberal in unattended (high $\sigma$ ) compared to the attended (low $\sigma$ ) conditions. There are, however, many other relationships that could equally satisfy this inequality-for example, an observer might scale their criterion by the square root of the variance (orange, panel A). This would similarly account for 
the empirical finding of a monotonically decreasing (i.e. more liberal) relative decision criterion in a high- $\sigma$ compared to a low$\sigma$ condition (orange curve, panel B). Lastly, the Bayes-optimal solution entails optimal sensitivity of the absolute decision vari- able $k$ as $\sigma$ increases (green line, panel A), which corresponds to a fixed relative criterion in a $\sigma$-normalized space (green line, panel B). 
$\mathrm{A}$

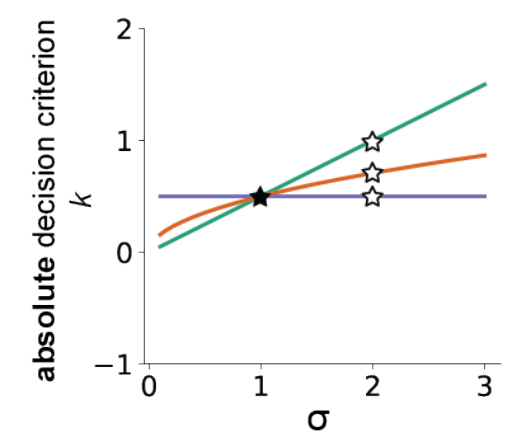

C

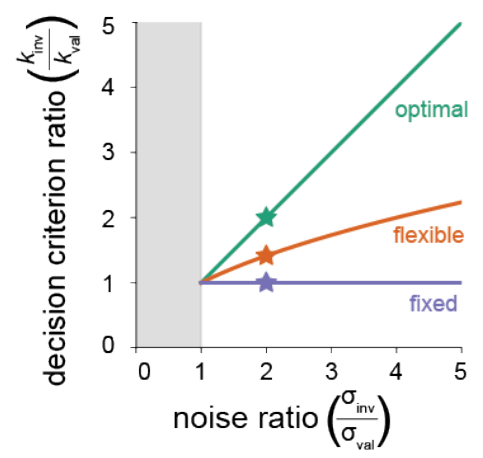

B

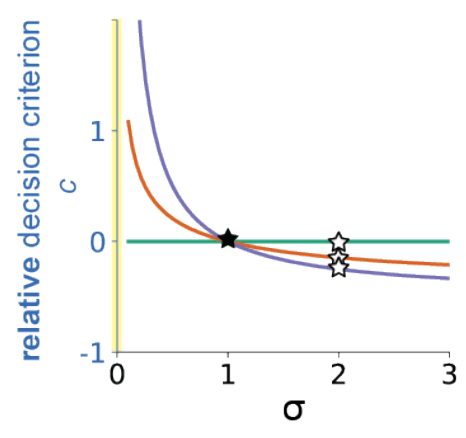

D

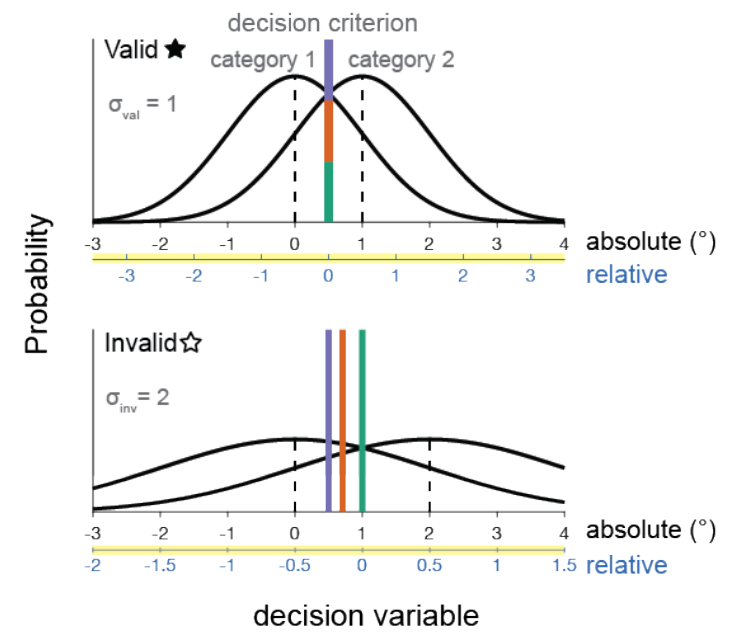

Figure 1: Three possible relationships between the trial-to-trial variance of the decision variable $\sigma$ and an observer's absolute (A) or relative (B) decision criterion. On a detection or coarse discrimination task, we assume that inattention leads to an increase in the trial-to-trial variability of the decision variable (i.e., an increase in $\sigma$ from valid (filled star) to invalid (open star) conditions. Here, we assume an increase from $\sigma_{\text {val }}=1$ to $\sigma_{\text {inv }}=2$ (where the decision variable distributions across conditions are pictured in panel D). Adherents to the fixed criterion assumption believe that the absolute criterion remains fixed as $\sigma$ increases (purple line, panel A). This corresponds to a decreasing relative criterion (i.e., a more "liberal" relative criterion) as variability increases (purple curve, panel B). An observer might alternatively scale their criterion by the square root of the variance (orange, panel A). This would similarly account for the empirical finding of a monotonically decreasing (i.e. more liberal) relative decision criterion in a high- $\sigma$ compared to a low- $\sigma$ condition (orange curve, panel B). Lastly, the Bayes-optimal solution (Appendix A) entails optimal sensitivity of the absolute decision variable $k$ as $\sigma$ increases (green line, panel A), which corresponds to a fixed relative criterion in a $\sigma$-normalized space (green line, panel B).

(C) Visualizing the space of possible absolute criterion and decision variable variance ratios that satisfy the IARF. Any combination of parameters below the green optimal line would satisfy the IARF. The fixed criterion observer posited by Rahnev (purple) is given by the purple line with a fixed criterion ratio of 1 . Any point above the purple line would violate the fixed criterion assumption. An observer who scales their measurement criterion by the square root of the noise level (orange) also satisfies the IARF and serves as just one of many possible examples of suboptimal, non-fixed models that do so. Anything between the opt line and fixed line suggests a shifting criterion in the correct direction which falls short of the optimal magitude. The grey zone represents combinations where invalid noise is smaller than valid noise, which we assume is not possible. The zone below the fixed line, where invalid criterion is lower than the valid criterion, while unlikely, would still be consistent with the IARF.

(D) Shifts in the decision criteria from valid (top) to invalid (bottom) conditions in an example case where $\sigma_{\text {val }}=1$ and $\sigma_{\text {inv }}=2$. Decision variable distributions are given by the black curves. The decision criteria used to separate category 1 from category 2 are given by vertical lines of their respective colours. The decision variable distributions and decision criteria can be expressed in a normalized, relative space (lower $\mathrm{x}$-axis), in which the Bayes-optimal decision criterion is fixed at 0 . They can also be expressed in an absolute decision variable space, which we assume to be equal to degrees in a coarse discrimination task. In absolute decision variable space, the fixed criterion model suggests a decision criterion (purple) which stays fixed from valid to invalid conditions (resulting in a relative decision criterion which is said to shift leftward). Here we show a fixed criterion observer which happens to be optimal in the valid condition, but those observers which set $\$$ single suboptimal criterion for both valid and invalid conditions is also fixed. 
From (19), we visualize the space of possible absolute criterion and decision variable variance ratios that satisfy the IARF in [Figure 1c]. Any combination of parameters below the green opt line would satisfy the IARF. The fixed criterion observer posited by Rahnev is given by the purple line, with a fixed criterion ratio of 1 . Any point above the purple line would violate the unified criterion assumption. An observer who scales their measurement criterion by the square root of the noise level (orange line) also satisfies the IARF and serves as just one of many possible examples of suboptimal, non-fixed models that do so. Anything between the opt line and fixed line suggests a shift in the criterion in the correct direction, but one which falls short of the optimal magnitude.

We should moreover leave open the possibility that even a fully Bayesian observer who has wrong beliefs about certain features of the generative model can also satisfy the IARF. We demonstrate this possibility in Appendix B.

\section{Tasks that distinguish between a fixed and a flexible criterion}

Since we are interested in distinguishing between a fixed and flexible criterion in observers, the goal is to determine and compare the values of $k_{\text {val }}$ and $k_{\text {inv }}$. But as we have demonstrated above, finding a unique solution for $k$ is non-trivial on most task designs. We propose that two experimental requirements must be met when testing the fixed-criterion hypothesis: Firstly, the decision variable stimulus must be plausibly identifiable with some known stimulus-derived feature axis. Secondly, the task design must be such that subjects have an incentive to shift their criterion as uncertainty changes. We will now elaborate on these requirements.

\section{A. Requirement 1: A determinate mapping from the stimulus space to the decision variable space}

We propose that the problem we ran into in the previous section is an example of a more general problem with standard signal detection theory paradigms, which we call the "indeterminate mapping" problem. The problem is that, in most signal detection theoretic tasks, we do not know how an observer maps the stimulus variable space that the experimenter can access and manipulate (orientation, contrast, luminance) to the observer's own internal decision variable space, in order to make decisions in the task at hand. The observer's decision variable space may reflect some non-linear warping of the stimulus variable space of interest, or may reflect a different stimulus variable space altogether than what the experimenter had in mind. For instance, an experimenter may manipulate units of contrast in a Gabor detection task, but the observer's decision variable might instead reflect units of luminance for a given patch of pixels. Even more subtly, the observer's decision variable may scale with contrast but in some non-linearly compressive way, for example, obeying a relationship characterized by Weber's law, whereby a unit increase in a stimulus property like contrast or luminance may result in a supra or infra-linear increase in an observer's decision variable, depending on the absolute magnitude of the property. Such examples illustrate the need to carefully rule out plausible alternatives before making conclusions about what physical properties of the stimulus subjects use as the basis for their decision variable. We cannot pin down the trial-to-trial mean of an observer's decision variable $(\mu)$ unless we are able to justify our assumption about the observer's mapping from stimulus feature to decision variable.

We run into the problem of a degeneracy of criterion solutions for $k$ as a result of our inability to pin down a definite value for $\mu$.

Recall that Eq. (6)

$$
c=\frac{k-\frac{\mu}{2}}{\sigma}
$$

shows that $c$ is the distance between the measurement criterion and the optimal criterion, also expressed in units of standard deviation. Likewise, Eq. (7)

$$
d^{\prime}=\frac{\mu}{\sigma}
$$

shows that $d^{\prime}$ is the distance between the means of the signal and noise distributions, in units of standard deviation. The two relative quantities $d^{\prime}$ and $c$, which we can measure in an experiment, are therefore expressed in terms of three absolute quantities $\mu, \sigma$, and $k$, which we would like to infer. We cannot infer three variables from two measurements, so if we do not know (or cannot plausibly assume to know) either $\mu$ or $\sigma, k$ will remain fundamentally unidentifiable, leading to the indeterminacy problem described in the above section. (See Denison et al, 2018 [17], Appendix 1).

Thus, in order to pin down the decision criterion $k$, we must first pin down the mean of the decision variable $\mu$. And in order to pin down $\mu$, we need to design a task that allows us to plausibly assume that $\mu$ is identical to some stimulus feature. This requirement is not met by the standard stimulus detection task exemplified by Rahnev 2011 because we do not know the mapping between the experimenter-set contrast and the observer's internal decision variable for detection. Importantly, we have no way of knowing how attention changes the decision variable. Therefore, on detection tasks, we cannot say how changes in either physical stimulus contrast or attention lead, over many trials, to changes in $\mu$ or $\sigma$ in decision variable space.

Simple orientation discrimination tasks (that is, for example, coarse discrimination between $-45^{\circ}$ and $45^{\circ}$ or fine discrimination between $-2^{\circ}$ and $2^{\circ}$ ) also fall victim to the mapping problem because there are alternative decision variable axes that subjects may plausibly use than orientation. On the possibility that the subject's decision variable is faithful to orientation, each unit on the decision variable axis would correspond to a different orientation in degrees (where the half-way point between $-45^{\circ}$ and $45^{\circ}$ is an orientation of $0^{\circ}$ ). This could be implemented by reading the difference in firing rates between two neural populations, 
each of which is maximally responsive to the stimulus orientation corresponding to the one of the two stimulus values. In general however, we are unable to rule out any such scheme that involves a decision variable which is low at one extreme (corresponding to stimulus 1), high at the other extreme (corresponding to stimulus 2), and monotonically increasing. And any such axis could be implemented by the difference in firing rates between two neural populations $(\Delta r)$, where each population fires maximally for a their preferred stimulus feature. For example, an alternative possibility to orientation is that the subject's decision variable reflects a graded linear interpolation in pixel space between $-45^{\circ}$ and $45^{\circ}$ (where the half-way point in the decision variable space reflects an equal superimposition of an image of $-45^{\circ}$ and $45^{\circ}$ ), with two populations maximally sensitive to either extreme. In simple discrimination tasks, we are unable to rule out the former scenario in favor of the latter, nor are we able to rule out nonlinear variations of either scenario that preserve a monotonic mapping.

We believe the most promising approach for encouraging observers to use a particular axis is to present a continuum of stimuli along the desired decision variable axis and to ask subjects to categorize the stimuli into one of two learned category distributions. For example, subjects could categorize an oriented Gabor as drawn from one of two overlapping normal distributions with different means (e.g., $+/-5^{\circ}$ ) and the same standard deviation (e.g., $8^{\circ}$ ). In requiring subjects to distinguish between distributions of orientations (and not merely two fixed orientations, as in traditional discrimination tasks), these tasks provide stronger justification for the assumption that the decision variable is in fact identical to orientation, since orientation is the simplest plausible variable that accurately reflects strength of evidence for category delineation.

In sum, we propose that we can only pin down $\mu$ if we can assert a one-to-one mapping between a known physical stimulus space and an unobservable decision variable space. It is important to seek a task design where subjects are as limited as possible to using only the decision variable mapping intended by the experimenter. One way to achieve this is by presenting a continuum of stimuli along the desired decision variable axis and asking subjects to identify stimuli as belonging to different distributions along this axis.

\section{B. Requirement 2: Built-in incentive to shift the decision crite- rion based on uncertainty}

Secondly, even if we had full knowledge of the mapping between a stimulus feature and the subject's decision variable, an additional condition for resolving the effect of uncertainty on the decision criterion is that the task must build in some incentive to move one's decision criterion according to uncertainty. For instance, in any discrimination or categorization task in which the category distributions are symmetric and offset (e.g., the internal measurement distributions for the two categories have the same variance and means $+/-\mu$ ), the optimal decision variable is at the intersection point of the two internal measurement distributions. This point stays the same as uncertainty changes (Figure 2) panel A, so observers have no incentive to shift their decision criterion. In this situation, we would not expect differences in $k_{\text {inv }}$ and $k_{\text {val }}$ from an optimal observer. (See Denison et al, 2018 [17], Appendix 1.) Thus, to study whether uncertainty affects decision criteria, a task should provide an optimal observer with an incentive to shift their decision criterion when uncertainty changes.

\section{The embedded category task}

The "embedded category" task (Figure 2, panel B; Qamar et al., 2013 [14]; Denison et al., 2018 [17]; Adler et al., 2018 [20]) meets both criteria for distinguishing between a fixed and flexible criterion. To infer attention-dependent shifts in $k$ based on measurements of $d^{\prime}$ and $c$, we have outlined that it is necessary to know either $\mu$ or $\sigma$, and to provide a task structure that incentivizes observers to respond to increasing uncertainty by shifting $k$ - the embedded category task succeeds on both counts.

In the embedded category task, observers categorize a stimulus drawn from one of two distributions (categories) with the same mean but different standard deviations (Figure 2, panel B). For example, in an orientation categorization version of this task, observers would categorize a given orientation as drawn from either a narrow distribution (category 1) or a broad distribution (category 2), both centered around horizontal (0 degrees). When the orientation of the test stimulus is near horizontal, it is more likely to belong to category 1 , whereas when the orientation is far from horizontal, it is more likely to belong to category 2 . 

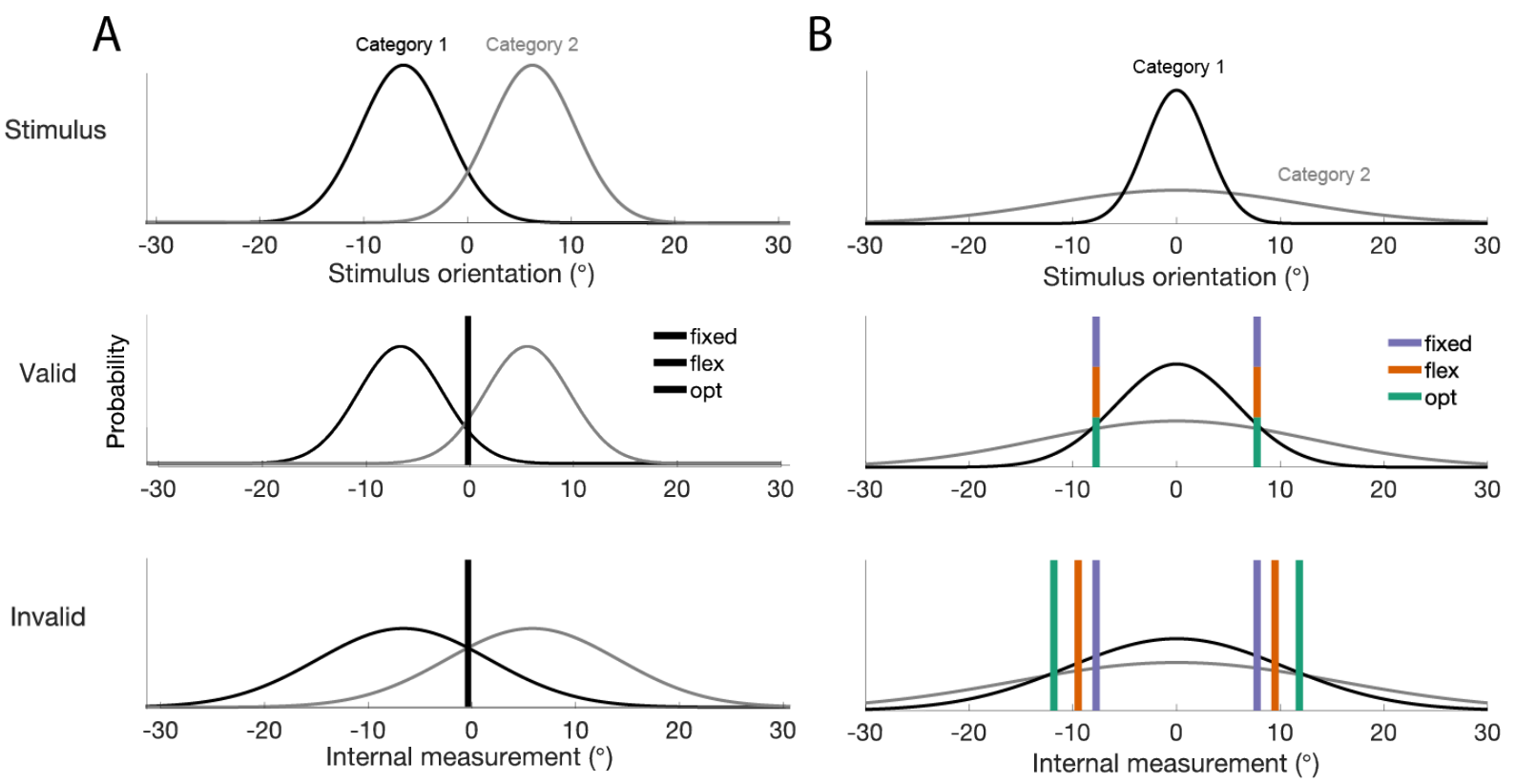

Figure 2: (A): Standard signal detection theoretic task. For discrimination or categorization tasks in which the category distributions are symmetric and offset (e.g., where category 1 produces an internal decision variable distribution with mean $-\mu$ and variance $\sigma$ and where category 2 produces a distribution of mean $\mu$ and equal variance $\sigma$ ), there is typically no incentive for the optimal observer to shift their decision criterion as trial-to-trial variability increases. The optimal decision criterion continues to bisect the means of the two category distributions, thus failing to provide an optimal observer with an incentive to shift their decision criterion as uncertainty changes. (B): The embedded category task. In a variant of the standard fine-discrimination task, observers categorize an oriented stimulus drawn from one of two orientation distributions: a narrow distribution (category 1) or a broad distribution (category 2), both centered around horizontal (0 degrees), such that when the orientation of the stimulus is near horizontal, it is more likely to be from category 1 . As variance increases from the valid to invalid condition, decision criteria may stay fixed (purple), adjust flexibly (e.g., square root relation, orange), or adjust optimally (green).

This task structure avoids the indeterminate mapping problem problem because orientation is the only plausible decision variable-it varies continuously from trial to trial and serves as the sole basis for category delineation. Since $\mu$ is known in physical units, and since $\mu$ is not expected to change when we manipulate the uncertainty of the stimulus by changing its physical contrast (Qamar et al., 2013 [14]; Adler et al., 2018 [20]) or level of attention (Denison et al., 2018) [17], it is therefore possible for us to infer the absolute criterion $k$ from task performance.

Moreover, the embedded category task, by virtue of having unequal category stimulus variance, incentivizes subjects to shift their decision boundary as measurement uncertainty increases (see Figure 2, panel B). The optimal decision boundary should shift with changes in uncertainty, satisfying our second requirement (Figure 2, panel B, green line). Therefore, by plausibly fixing $\mu$ and $\sigma$, and by incentivizing an uncertainty-dependent decision boundary, Denison et al. satisfy both minimum requirements for resolving the question of whether the absolute criterion, $k$, is fixed (Figure 2, panel B, purple line) or flexible (Figure 2, panel B, green line).

To briefly summarize Denison et al.'s results, they find that $k$ was not fixed but instead adjusted flexibly based on level of uncertainty, in line with previous findings that the decision criterion is broadly sensitive to uncertainty (Qamar et al., 2013 [14]; Denison et al., 2018 [17]; Adler et al., 2018 [20]). [see Denison et al., 2018, figure 4]

Similar results have been found in peripheral viewing tasks. For instance, Zhou et al., 2018 [12] asked subjects to make decisions about the co-linearity of horizontal lines at varying peripheral eccentricities (i.e. at varying degrees of sensory uncertainty). In line with much of the probabilistic perception literature, the study suggests that subjects perform nearly optimally in setting decision criteria to correspond to each level of visual eccentricity (i.e. each level of uncertainty), suggesting that uncertainty-dependent criterion-setting may also be involved in subjective inflation of visual perception at the periphery. This poses a challenge to Solovey et al., 2015, in which the authors only put forward a fixed criterion model to explain the phenomenon [4]).

\section{Other potential designs}

The embedded category task is far from the only experimental design that can meet the two task requirements to test whether decision criteria are sensitive to attention-dependent uncertainty 
(or other forms of uncertainty more broadly). The question can be answered by modifying existing experimental designs. For example, an auditory-visual cue combination task in which subjects are asked to report the location of an object, and in which uncertainty is manipulated via valid or invalid cuing, should help shed further light on the question of flexible criteria. This is because, unlike a standard 2AFC detection or coarse discrimination task, the uncertainty manipulation should map to the observer's decision variable space in a fairly predictable way (i.e., we should not expect inattention to systematically warp the observer's internal decision space if that decision variable reflects spatial location), and because unequal measurement variance between the sensory modalities is expected to result in a shift in criterion as uncertainty is manipulated. Here, the unequal measurement variance caused by differences in reliability between visual and auditory information would play an analogous role to the unequal category variances in the embedded category task. Cue combination tasks typically involve estimates over a continuous variable (e.g. location), but can be converted into a decision-making task through comparison of that estimate to a standard cue (e.g. is the stimulus to the left, right, or straight ahead, relative to a standard cue?). There is a paucity of research in the cue combination domain that manipulates uncertainty through attention rather than a stimulus-driven manipulation (e.g. manipulations cue reliability). Cue combination tasks with an attentional manipulation paired with neural recording can also help shed light on the neural underpinnings of criterion-setting (for instance, a variant of [21]). Additionally, a number of studies in which subjects are shown to use trial-to-trial sensory uncertainty information without trial-to-trial feedback may be suitable for modification to probe the effects of attention-dependent uncertainty on observer estimates and decision criteria (for more discussion of suitable tasks that may be adapted, see [22]).

Introducing unequal rewards between the two categories could also introduce an incentive to shift the decision criterion under increased uncertainty. The basic design could be similar to Whiteley and Sahani (2008) [23], in which observers did a leftright categorization task under unequal rewards for left and right. Rather than manipulating reward, one could fix the rewards for either choice (while keeping them unequal) and manipulate attention. If attention acts only on the sensory noise level $\sigma$, then the reward-maximizing criterion will be attention-dependent. The same effect would be obtained by assigning different base rates (prior probabilities) to the two categories [24]. However, these manipulations introduce an extra ingredient and further assumptions compared to the embedded category task. In these tasks, observers need to both learn the prior or reward information and combine that information with the likelihood. Only then would they have the potential to appropriately adjust their decision criterion as a function of attention-dependent uncertainty.

\section{Conclusion}

In this paper, we have investigated an apparent discrepancy in two parallel lines of research- research in the field of conscious perception and inattentional inflation have proposed that a subject's decision criterion is a fixed threshold which does not adjust based on level of attention, while research in the field of perceptual decision-making suggests that the criterion is a flexible boundary which is sensitive to attention-dependent uncertainty. We have shown that current empirical evidence does not support the adoption of a "fixed criterion" assumption. Both fixed and flexible criteria are consistent with Rahnev et al.'s empirical findings, and indeed an infinite set of relations between the observer's trialto-trial decision variable and decision criterion could account for these results. Moreover, we have shown that not all task designs are suitable for demonstrating flexible criterion-setting because multiple plausible decision variable mappings may exist for a given task. Using an embedded category task design which allows for the presentation of intermediate stimuli along the desired feature axis encourages observers to use orientation rather than any other stimulus feature as the basis for their decision variable. A distinct advantage of distribution-based categorization tasks is that we can make better assumptions about the $\mu$ and $\sigma$ of the observer's internal decision variable distributions to make better inferences about the absolute decision criterion $k$. Moreover, if we are interested in investigating the sensitivity of an observer's decision criterion to attention-dependent uncertainty, we should prefer experimental designs where there is an accuracy incentive for observers to shift their criterion as the trial-to-trial variance of their decision variable changes. Because Denison et al.'s embedded category task offers both distinct advantages over previous SDT experiments, we should take seriously the empirical finding that observers' decision rules are sensitive to attentiondependent uncertainty in that task, and incorporate that finding into future modelling assumptions when investigating perceptual inflation. This analysis suggests the criterion for conscious perception (and not only decision-making) may be sensitive to uncertainty caused by inattention or peripheral viewing. Therefore, an important future experimental direction is to develop a perceptual detection task that meets the requirements described in this paper and assess using this improved methodology whether perceptual criteria are fixed or flexible. 


\section{APPENDIX}

\section{A. Optimal Bayesian observer}

Under what condition is the IARF satisfied by a Bayesian observer? Bayesian models are a subset of signal detection theoretic models in which the criterion is not any arbitrary number, but is instead set to maximize expected accuracy (or another form of expected utility). A Bayesian observer uses particular pre-existing beliefs about the world to make inferences. If these beliefs are correct -i.e. reflect the true generative model of the observations - then expected accuracy will in fact be maximized. In the present section, we will treat that case; in the following section, we will allow for wrong beliefs.

Let's say that on a given trial, the measurement of the stimulus is $x_{\text {trial }}$. The Bayesian observer uses knowledge of the generative model defined by Equations (1) and (2) to make the best possible judgment about the stimulus $s$ given $x_{\text {trial }}$. The "best possible" judgement here means the one that will maximize accuracy over many trials. This requires choosing the category ("present", $s=\mu$ or "absent", $s=0$ ) with the highest posterior probability. To formalize this, we define the Bayesian observer's internal decision variable $d$ as the log posterior ratio

$$
d \equiv \log \frac{p\left(s=\mu \mid x_{\text {trial }}\right)}{p\left(s=0 \mid x_{\text {trial }}\right)}
$$

This can be written as the sum of the log likelihood ratio and the log prior ratio:

$$
d=\log \frac{p\left(x_{\text {trial }} \mid s=\mu\right)}{p\left(x_{\text {trial }} \mid s=0\right)}+\log \frac{p(s=\mu)}{p(s=0)} .
$$

Substituting Eqs. (1) and (2), we find

$$
d=\frac{\mu\left(x_{\text {trial }}-\frac{\mu}{2}\right)}{\sigma^{2}} .
$$

Reporting the stimulus with the highest posterior probability means reporting "present" when $d>0$, or in other words, when $x_{\text {trial }}>\frac{\mu}{2}$. Comparing this with Eq. (5), we find that the optimal Bayesian criterion is

$$
k_{\mathrm{opt}}=\frac{\mu}{2} .
$$

We now apply this equation to the comparison between valid and invalid conditions:

$$
k_{\mathrm{opt}, \text { inv }}=\frac{\mu_{\mathrm{opt}, \mathrm{inv}}}{\mu_{\mathrm{opt}, \mathrm{val}}} k_{\mathrm{opt}, \mathrm{val}} .
$$

Since experimenters ensure that $d^{\prime}$ is matched between the valid and invalid conditions, this is equivalent to

$$
k_{\mathrm{opt}, \text { inv }}=\frac{\sigma_{\mathrm{opt}, \text { inv }}}{\sigma_{\mathrm{opt}, \mathrm{val}}} k_{\mathrm{opt}, \mathrm{val}} .
$$

This does not satisfy the IARF, Eq. (19), since that is a strict inequality. Stated plainly, Rahnev's empirical findings suggest that people are not Bayes-optimal in the task, which the authors rightly emphasize. As measurement noise increases, observers do not adjust their decision criterion by the Bayes-optimal amount- adjustments fall short of this magnitude.

Additionally, we can easily rule out another general class of suboptimal models: If $k=\alpha \mu$ or $k=\alpha \sigma$, where $\alpha$ is an arbitrary constant, then the IARF is also not satisfied.

\section{B. Bayesian observer with wrong beliefs}

So far, we have considered an optimal Bayesian observer who holds correct beliefs about the generative model. While such an observer is not consistent with the data, a Bayesian observer who holds incorrect beliefs about the generative model might still be. Such an observer is said to have model mismatch - their internal model of the world is mismatched in some way to the task's true generative model. A Bayesian observer with model mismatch will of course be, strictly speaking, "suboptimal." Suboptimalities in inference have been proposed to account for variability in human behavior in other contexts [25, 26, 13]. Specifically, we allow here that an observer may have wrong beliefs about $\mu, \sigma$, and $\pi$, the prior probability that the stimulus is present. We denote the observer's belief about the stimulus value when the stimulus is present by $\tilde{\mu}$, their belief about noise level by $\tilde{\sigma}$, and their belief about the probability that the stimulus is present by $\tilde{\pi}$. Then, Eq. (25) for the log posterior ratio $(d)$ becomes

$$
d=\frac{\tilde{\mu}\left(x_{\text {trial }}-\frac{\tilde{\mu}}{2}\right)}{\tilde{\sigma}^{2}}+\mathrm{LPR}, .
$$


where we define the believed log prior ratio LPR as

$$
\operatorname{LPR} \equiv \log \frac{\tilde{\pi}}{1-\tilde{\pi}} .
$$

The decision rule of responding "present" when $d>0$ now becomes

$$
x_{\text {trial }}>\frac{\tilde{\mu}}{2}+\frac{\tilde{\sigma}^{2}}{\tilde{\mu}} \mathrm{LPR},
$$

Comparing with Eq. (5), we see that the Bayesian criterion is

$$
k=\frac{\tilde{\mu}}{2}+\frac{\tilde{\sigma}^{2}}{\tilde{\mu}} \mathrm{LPR}
$$

Eq. (33) indicates that for the Bayesian observer, the criterion is not free but is fully determined by the parameters that describe the observer's beliefs over the structure of the world. The invalid and valid Bayesian criteria are

$$
\begin{aligned}
& k_{\text {Bayes, inv }}=\frac{\tilde{\mu}_{\text {inv }}}{2}+\frac{\tilde{\sigma}_{\text {inv }}^{2}}{\tilde{\mu}_{\text {inv }}} \mathrm{LPR} \\
& k_{\text {Bayes, val }}=\frac{\tilde{\mu}_{\text {val }}}{2}+\frac{\tilde{\sigma}_{\text {val }}^{2}}{\tilde{\mu}_{\text {val }}} \mathrm{LPR}
\end{aligned}
$$

These expressions can be rewritten as

$$
\begin{aligned}
& k_{\text {Bayes, inv }}=\tilde{\sigma}_{\text {inv }}\left(\frac{\tilde{d}_{\text {inv }}^{\prime}}{2}-\frac{\mathrm{LPR}}{\tilde{d}_{\text {inv }}^{\prime}}\right) \\
& k_{\text {Bayes, val }}=\tilde{\sigma}_{\text {val }}\left(\frac{\tilde{d}_{\text {val }}^{\prime}}{2}-\frac{\mathrm{LPR}}{\tilde{d}_{\text {val }}^{\prime}}\right)
\end{aligned}
$$

where we defined the believed sensitivities

$$
\begin{aligned}
& \tilde{d}_{\mathrm{val}}^{\prime} \equiv \frac{\tilde{\mu}_{\mathrm{val}}}{\tilde{\sigma}_{\mathrm{val}}} \\
& \tilde{d}_{\mathrm{inv}}^{\prime} \equiv \frac{\tilde{\mu}_{\mathrm{inv}}}{\tilde{\sigma}_{\mathrm{inv}}} .
\end{aligned}
$$

We now examine the circumstances under which a Bayesian observer with wrong beliefs sets criteria that satisfy the IARF, Eq. (19). For such an observer, the IARF can be rewritten as

$$
\frac{\tilde{\sigma}_{\text {inv }}}{\sigma_{\text {inv }}}\left(\frac{\tilde{d}_{\text {inv }}^{\prime}}{2}-\frac{\text { LPR }}{\tilde{d}_{\text {inv }}^{\prime}}\right)<\frac{\tilde{\sigma}_{\text {val }}}{\sigma_{\text {val }}}\left(\frac{\tilde{d}_{\text {val }}^{\prime}}{2}-\frac{\text { LPR }}{\tilde{d}_{\text {val }}^{\prime}}\right) .
$$

There are many combinations of $\tilde{\pi}, \tilde{d}_{\text {inv }}^{\prime}, \tilde{d}_{\text {val }}^{\prime}, \tilde{\sigma}_{\text {inv }}^{\prime}$, and $\tilde{\sigma}_{\text {val }}^{\prime}$ for which Eq. (40) holds. A few special cases are of interest.

Special Case 1. Firstly, if the observer uses the correct $\sigma$ s and the correct value of the prior, $\tilde{\pi}=0.5$, then LPR $=0$ and Eq. (40) becomes

$$
\tilde{d}_{\mathrm{val}}^{\prime}>\tilde{d}_{\mathrm{inv}}^{\prime}
$$

Thus, wrong beliefs about sensitivity could be solely responsible for Rahnev's findings. Even though experimenters matched $d^{\prime}$ across conditions, it may be the case that subjects incorrectly believe their sensitivity to be higher for the valid compared to the invalid conditions.

Special Case 2. Secondly, if the observer correctly believes that $\tilde{d}_{\mathrm{val}}^{\prime}=\tilde{d}_{\mathrm{inv}}^{\prime}$, but holds incorrect beliefs about either or both $\sigma \mathrm{s}$, then Eq. (40) reduces to

$$
\frac{\tilde{\sigma}_{\text {inv }}}{\sigma_{\text {inv }}}<\frac{\tilde{\sigma}_{\text {val }}}{\sigma_{\text {val }}}
$$


This means that the noise level in the valid condition (the lower noise level) is overestimated by a greater factor relative to its true value than the noise level in the invalid condition. (Or, equivalently, the noise level in the invalid condition is underestimated by a greater factor than the noise level in the valid condition.) Thus, wrong beliefs about internal noise in either or both conditions could also be solely responsible for Rahnev's findings.

Special Case 3. Thirdly, if the observer uses the correct $\sigma \mathrm{s}$ but $\tilde{\pi}$ is not necessarily equal to 0.5 , and they incorrectly believe that $\tilde{d}_{\text {val }}^{\prime}>\tilde{d}_{\text {inv }}^{\prime}$, then Eq. (40) becomes

$$
\mathrm{LPR}<\frac{\tilde{d}_{{ }_{\text {val }}^{\prime}} \tilde{d}_{\text {inv }}}{2} .
$$

Since $d^{\prime}$ s are positive, and since $\tilde{\pi} \leq 0.5$ results in a LPR $\leq 0$, this condition is always satisfied when $\tilde{\pi} \leq 0.5$. However, it also holds for higher values of $\tilde{\pi}$, up to a limit determined by the believed $d^{\prime}$ s. An analogous scenario with opposite signs arises when the observer uses the correct $\sigma$ s but incorrectly believes that $\tilde{d}_{\text {val }}^{\prime}<\tilde{d}_{\text {inv }}^{\prime}$.

We conclude, firstly, that within the Bayesian framework, there are multiple sets of wrong beliefs that can account for Rahnev's findings without positing a fixed decision criterion. Secondly, even within the Bayesian framework, Rahnev's findings do not imply that the criterion is the same between the invalid and valid conditions.

\section{References}

[1] Dobromir Rahnev et al. "Attention induces conservative subjective biases in visual perception". In: Nature neuroscience 14.12 (2011), p. 1513.

[2] Hakwan Lau and David Rosenthal. "Empirical support for higher-order theories of conscious awareness". In: Trends in cognitive sciences 15.8 (2011), pp. 365-373.

[3] Brian Odegaard et al. "Inflation versus filling-in: why we feel we see more than we actually do in peripheral vision". In: Philosophical Transactions of the Royal Society B: Biological Sciences 373.1755 (2018), p. 20170345.

[4] Guillermo Solovey, Guy Gerard Graney, and Hakwan Lau. "A decisional account of subjective inflation of visual perception at the periphery". In: Attention, Perception, \& Psychophysics 77.1 (2015), pp. 258-271.

[5] Luigi Acerbi, Sethu Vijayakumar, and Daniel M Wolpert. "On the origins of suboptimality in human probabilistic inference". In: PLoS Comput Biol 10.6 (2014), e1003661.

[6] Konrad P Körding and Daniel M Wolpert. "Bayesian integration in sensorimotor learning”. In: Nature 427.6971 (2004), pp. 244-247.

[7] Mehrdad Jazayeri and Michael N Shadlen. "Temporal context calibrates interval timing". In: Nature neuroscience 13.8 (2010), p. 1020.

[8] David Alais and David Burr. "The ventriloquist effect results from near-optimal bimodal integration”. In: Current biology 14.3 (2004), pp. 257-262.

[9] Konrad P Körding et al. "Causal inference in multisensory perception”. In: PLoS one 2.9 (2007), e943.

[10] Robert J Van Beers, Anne C Sittig, and Jan J Denier van der Gon. "Integration of proprioceptive and visual positioninformation: An experimentally supported model”. In: Journal of neurophysiology 81.3 (1999), pp. $1355-1364$.

[11] David C Knill and Jeffrey A Saunders. "Do humans optimally integrate stereo and texture information for judgments of surface slant?” In: Vision research 43.24 (2003), pp. 2539-2558.

[12] Yanli Zhou, Luigi Acerbi, and Wei Ji Ma. “The Role of Sensory Uncertainty in Simple Perceptual Organization”. In: bioRxiv (2018), p. 350082.

[13] Shan Shen and Wei Ji Ma. "A detailed comparison of optimality and simplicity in perceptual decision making." In: Psychological review 123.4 (2016), p. 452.

[14] Ahmad T Qamar et al. "Trial-to-trial, uncertainty-based adjustment of decision boundaries in visual categorization". In: Proceedings of the National Academy of Sciences 110.50 (2013), pp. 20332-20337.

[15] Wei Ji Ma et al. "Behavior and neural basis of near-optimal visual search". In: Nature neuroscience 14.6 (2011), p. 783.

[16] Scott Cheng-Hsin Yang, Mate Lengyel, and Daniel M Wolpert. "Active sensing in the categorization of visual patterns". In: Elife 5 (2016), e12215. 
[17] Rachel N Denison et al. "Humans incorporate attention-dependent uncertainty into perceptual decisions and confidence". In: Proceedings of the National Academy of Sciences 115.43 (2018), pp. 11090-11095.

[18] DM Green and JA Swets. "Signal detection theory and psychophysics (Rev. ed.)" In: Huntington, NY: RF Krieger (1974).

[19] Andrei Gorea and Dov Sagi. "Failure to handle more than one internal representation in visual detection tasks". In: Proceedings of the National Academy of Sciences 97.22 (2000), pp. 12380-12384.

[20] William T Adler and Wei Ji Ma. "Comparing Bayesian and non-Bayesian accounts of human confidence reports". In: PLoS computational biology 14.11 (2018), e1006572.

[21] Yong Gu, Dora E Angelaki, and Gregory C DeAngelis. "Neural correlates of multisensory cue integration in macaque MSTd". In: Nature neuroscience 11.10 (2008), pp. 1201-1210.

[22] Wei Ji Ma and Mehrdad Jazayeri. "Neural coding of uncertainty and probability". In: Annual review of neuroscience 37 (2014), pp. 205-220.

[23] Louise Whiteley and Maneesh Sahani. "Implicit knowledge of visual uncertainty guides decisions with asymmetric outcomes". In: Journal of vision 8.3 (2008), pp. 2-2.

[24] Jorge Morales et al. "Low attention impairs optimal incorporation of prior knowledge in perceptual decisions". In: Attention, Perception, \& Psychophysics 77.6 (2015), pp. 2021-2036.

[25] Jeffrey M Beck et al. "Not noisy, just wrong: the role of suboptimal inference in behavioral variability". In: Neuron 74.1 (2012), pp. 30-39.

[26] Jan Drugowitsch et al. "Computational precision of mental inference as critical source of human choice suboptimality". In: Neuron 92.6 (2016), pp. 1398-1411. 